\title{
Review
}

\section{Caspase substrates}

\author{
JC Timmer ${ }^{1,2}$ and GS Salvesen ${ }^{\star 2}$
}

The relatively common occurrence of sequences within proteins that match the consensus substrate specificity of caspases in intracellular proteins suggests a multitude of substrates in vivo - somewhere in the order of several hundred in humans alone. Indeed, the list of proteins that are reported to be cleaved by caspases in vitro proliferates rapidly. However, only a few of these proteins have been rigorously established as biologically or pathologically relevant, bona fide substrates in vivo. Many of them probably simply represent 'innocent bystanders' or erroneous assignments. In this review we discuss concepts of caspase substrate recognition and specificity, give resources for the discovery and annotation of caspase substrates, and highlight some specific human or mouse proteins where there is strong evidence for biologic or pathologic relevance.

Cell Death and Differentiation (2007) 14, 66-72. doi:10.1038/sj.cdd.4402059; published online 3 November 2006

\section{What it Takes to be a Caspase}

There is scarcely a form of life - whether it be viral, microbial, plant or animal, that does not encode at least one protease. Indeed, because the ability to recycle scarce amino acids in early biotic environments would probably be crucial to survival, one can imagine that proteases were among the first proteins to appear in the most primitive replicating organisms. Scientists have been investigating proteolytic enzymes for over 150 years, working up the field of proteolysis to a high art, so that proteases became the best characterized diverse group of enzymes from both biochemical and structural perspectives. Proteases have long been used by biotechnologists in processes as diverse as the production of cheese to the cleaning of soiled clothing. And up until about 10 years ago most biologists thought of proteases as important mediators, or often annoying nuisances, that tended to degrade cellular proteins. Prokaryotes may use proteases mainly for degradation and recycling of proteins, but most of the 500-or-so mammalian proteases are involved in very limited and specific proteolytic events - initiating and transmitting signals - and a good example of this is the caspase family.

The name caspase derives from cysteine-dependent aspartate specific protease ${ }^{1}$ : catalysis is governed by a critical conserved Cys side chain of the enzyme, and by a stringent specificity for cleaving protein substrates containing Asp. The use of a Cys side chain as a nucleophile during peptide bond hydrolysis is common to several protease families. However, the primary specificity for Asp is very rare among proteases. Of the currently known human and mouse proteases only the caspase activator granzyme $\mathrm{B}$, a serine protease, shares this primary specificity. ${ }^{2,3}$ The primary specificity pockets of caspases are almost identical, being formed by the side chains of the strictly conserved residues, Arg-179, Arg-341 and Gln-283 (caspase-1 numbering convention). This deep, highly basic pocket (Figure 1) is perfectly shaped to accommodate an Asp side chain, accounting for the up to four orders of magnitude lower catalytic efficiency for cleavage of peptides with a $\mathrm{P}_{1}$ Glu residue in most caspases, ${ }^{4}$ with the notable exception of the Drosophila caspase Dronc, which seems to have an equal specificity for Asp and Glu. ${ }^{5}$ The caspases are members of a clan, or structurally related group, known as cysteine protease clan CD that also contains the plant metacaspases, ${ }^{6}$ and mammalian and plant proteases of the legumain family (involved in specific processing events), the eucaryotic protease separase (required for sister chromatid separation during mitosis), and the bacterial proteases clostripain and gingipain. ${ }^{7}$ Common to all these proteases is a shared stringent specificity for one type of amino acid side chain at the primary $\left(\mathrm{P}_{1}\right)$ specificity position in substrates (Arg or Lys for metacaspases, clostripain and gingipains; Asn for legumains; and Arg for separase). Thus, the caspase fold - the clan CD fold - supports unusually stringent specificity at the primary cleavage site. Distinguishing the caspases from each other are different requirements at the extended substrate recognition positions, described in Figure 2.

\section{What it Takes to be a Caspase Substrate}

Inherent specificity - synthetic substrates. There are principally two ways to investigate caspase substrates: (1)

${ }^{1}$ Graduate Program in Molecular Pathology, University of California San Diego, La Jolla, CA 92037, USA and ${ }^{2}$ Program in Apoptosis and Cell Death Research, The Burnham Institute for Medical Research, 10901 North Torrey Pines Road, La Jolla, CA 92037 USA

*Corresponding author: GS Salvesen, Program in Apoptosis and Cell Death Research, The Burnham Institute for Medical Research, 10901 North Torrey Pines Road, La Jolla, CA 92037, USA. Tel: +1 858646 3114; Fax: + 1858713 6274; E-mail: gsalvesen@burnham.org

Keywords: apoptosis; protease; synthetic substrate; substrate prediction

Abbreviations: APP, amyloid precursor protein; CAD, caspase-activated DNAse; HTT, Huntingtin; IAP, inhibitor of apoptosis protein; IBM, IAP binding motif; ICAD, inhibitor of CAD; PARP, poly(ADP-ribose) polymerase; RB, retinoblastoma-associated protein; Smac/DIABLO, second mitochondrial activator of caspases/direct IAP binding protein with low pl; TNFR, tumor necrosis factor receptor

Received 29.8.06; revised 09.10.06; accepted 09.10.06; Edited by S Kumar; published online 03.11 .06 
a

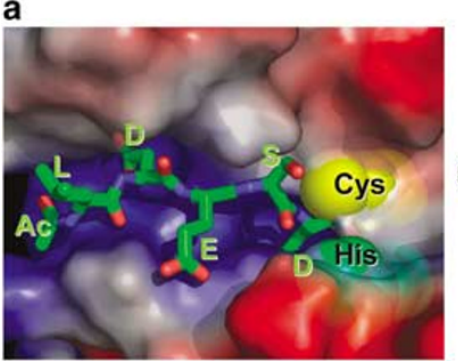

b

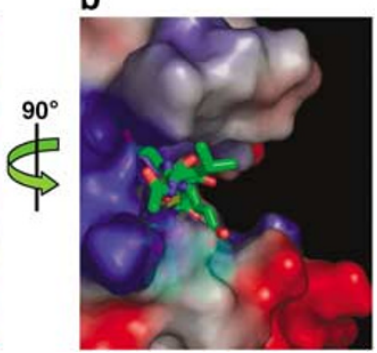

Figure 1 A caspase catalytic cleft. The active site cleft of caspase-2 bound to the peptapeptide inhibitor acetyl-Leu-Asp-Glu-Ser-Asp-aldehyde (Ac-LDESD$\mathrm{CHO}$ ), taken from PDB code $1 \mathrm{PYO} .^{68}$ The caspase is shown as a translucent electrostatic potential surface where blue hues denote positive potential and red hues negative potential. (a) The deep basic cleft next to the catalytic Cys (yellow) and His (green) residues of the enzyme occupies the $P_{1}$ Asp residue and is conserved in all active caspase structures. To the left of this are the $P_{2}-P_{5}$ residues, some occupying clearly visible specific pockets. The location of residues to the right (the $\mathrm{P}^{\prime}$ side) are unavailable in this or similar caspase structures because all common peptidyl inhibitors of caspases are designed without $\mathrm{P}^{\prime}$ substituents. (b) Rotation of the structure by $90^{\circ}$ about the $y$-axis reveals the mouth of the substrate cleft and the almost perfect extended geometry of the bound peptide. From this view it is clear that only extended protein strands will dock in a productive manner to the active site

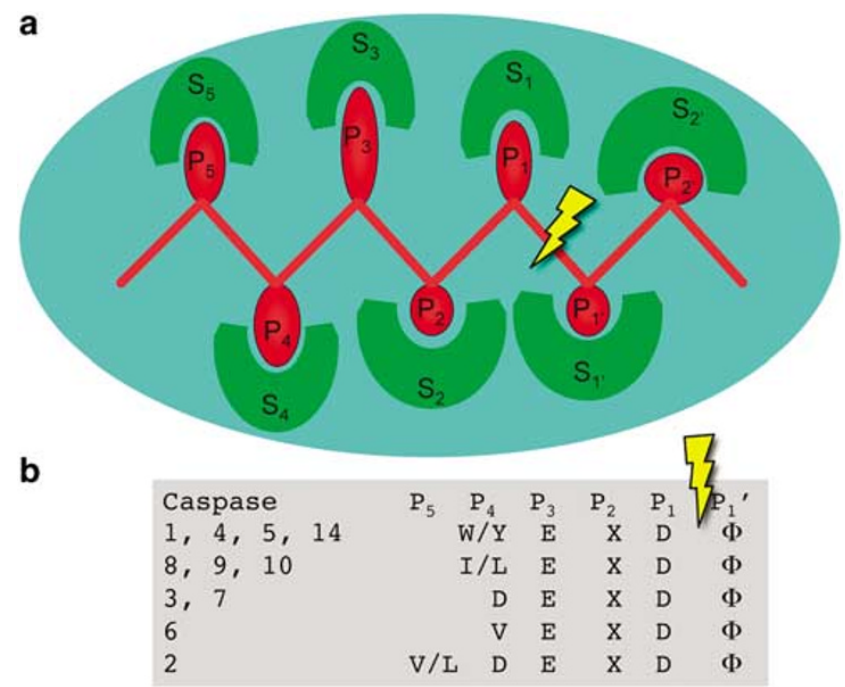

Figure 2 Inherent substrate specificity. The convention in naming substrate residues in protease active sites is to name the residue to the $\mathrm{N}$-terminal of the scissile bond (lightning bolt) $P_{1}$ and the one to the $\mathrm{C}$-terminal side $\mathrm{P}_{1}{ }_{1}$. (a) Other residues (red) are numbered consecutively from these origins. ${ }^{69}$ Many of these residues will occupy specific pockets on the enzyme, numbered with the matching ' $S$ ' designation (green), each of which may be constructed of several enzyme residues. The objective of this binding mode, which almost always binds the cleavage region in an extended peptide conformation, is to align the substrate accurately into register with the catalytic machinery. (b) Caspases bind substrates through typical interactions of the active site cleft with amino acids in the cleavage site. Studies with synthetic peptides and peptide libraries have shown that substrate residues $\mathrm{P}_{4}$ through $\mathrm{P}_{1}{ }^{\prime}$ contribute to binding through association with $\mathrm{S}_{4}$ through $\mathrm{S}_{1}{ }^{\prime}$ pockets in the active site. All caspases show exquisite selectivity for Asp in the $P_{1}$ position, a preference for Glu in $P_{3}$, and a lack of tolerance for charged residues at $\mathrm{P}_{1}{ }^{\prime}$ where Gly, Ala, Thr, Ser and Asn are preferred (denoted by the $\phi$ symbol). Distinguishing between them is primarily the nature of the $\mathrm{P}_{4}$ residue, and the inherent substrate specificities are generally well understood at the atomic level. Importantly caspase-2 has an additional interaction at $\mathrm{S}_{5}$ that enhances catalysis at least 30 -fold ${ }^{9}$

the inherent preferences in the active site cleft that distinguish them from each other, (2) their natural biologic substrates. The first tells us a lot about caspase catalysis and is much easier to investigate than the second. The seminal work of Thornberry et al. ${ }^{8}$ using positional scanning synthetic peptide-based libraries and Talanian et al. ${ }^{9}$ using sets of individual peptide substrates, later refined by others, led to our current understanding of the inherent substrate preferences summarized in Figure 2. From this came the idea of caspase-specific consensus recognition sequences that could be applied to predicting natural substrates. For example, many papers refer to the classic caspase-3 recognition consensus of DXXD $\downarrow$ (where $D$ is Asp, $X$ any amino acid, and $\downarrow$ denotes the site of cleavage). This is a vast oversimplification, and misses at least two important points. The first is that the residue after the scissile bond, known as $\mathrm{P}_{1}{ }^{\prime}$, is important in that charged or bulky residues are poorly tolerated. ${ }^{4}$ Gly, Ala, Thr, Ser and Asn are good, but Glu, Asp, Lys, Arg, Trp are very bad for catalysis. Thus, a good consensus would be DXXD $\downarrow$ G, for example, and a bad one would be DXXD $\downarrow E$. The second point is that it is dangerous to assume that a consensus is very meaningful per se for discovering natural substrates. We spend more time on this issue below, because it relates to the fundamental requirements that natural proteins have in order to be cleaved by proteases.

Knowledge of the inherent specificity of caspases in the $\mathrm{P}_{2}-\mathrm{P}_{4}$ positions has been employed by many investigators, as well as reagent companies, to construct peptide-based small substrate reporters that can be used to measure the activity of caspases. Such substrates have, typically, a tetrapeptide sequence matching a preferred caspase consensus coupled to a fluorogenic reporter that occupies the $P_{1}{ }^{\prime}$ position, and which is released to produce a fluorescent signal upon cleavage. Although these are useful in vitro to characterize individual purified caspases, they are virtually useless in distinguishing between caspases in complex milieu such as cell lysates. This is due to significant overlap between individual consensus sequences, because the caspases are promiscuous on these sequences, and because the amount of activity on substrates is highly dependent on the active caspase concentrations (which are difficult to calculate). However, the inherent cleavage site specificity of caspases have been applied to make active site probes that show some degree of selectivity for individual caspases, ${ }^{10}$ and when these are coupled with selective antisera the identity of active caspases in complex mixtures is more readily disclosed. ${ }^{11-14}$

\section{Specificity for protein substrates}

Exosites. Having a preferred cleavage site specificity is not sufficient for proteolysis of natural proteins; appropriate presentation of the cleavage site seems critical for efficient hydrolysis. Enzyme regions distant from the catalytic site that participate in substrate binding (exosites) are important determinants in the recognition of natural substrates by other regulatory proteases, such as those involved in blood coagulation $^{15}$ and matrix regulation. ${ }^{16}$ Additional interactions between caspases and substrates have been suggested, however, the mechanisms of these associations remain unclear. ${ }^{17}$ Currently, exosite interactions of caspases and 
Table 1 Comparison of models for predicting caspase substrates

\begin{tabular}{|c|c|c|c|c|c|}
\hline Reference & Specificity model $^{a}$ & $\begin{array}{l}\text { Cleavage } \\
\text { site range }\end{array}$ & Structural features & $\begin{array}{l}\text { Caspase } \\
\text { specificity }^{b}\end{array}$ & URL \\
\hline $\begin{array}{l}\text { BBFNN }^{42} \\
\text { CasPredictor }^{41} \\
\text { GrabCas }^{39}\end{array}$ & $\begin{array}{l}\text { Training set: } 13 \text { substrates } \\
\text { Training set: } 143 \text { substrates } \\
\text { Inherent specificity }\end{array}$ & $\begin{array}{l}\mathrm{P} 5-\mathrm{P} 5^{\prime} \\
\mathrm{P} 4-\mathrm{P} 1^{\prime} \\
\mathrm{P} 4-\mathrm{P} 1^{\prime}\end{array}$ & $\begin{array}{l}\text { No } \\
\text { PEST sequences } \\
\text { No }\end{array}$ & $\begin{array}{l}\text { pan-Caspase } \\
\text { pan-Caspase } \\
\text { Caspases } 1-9\end{array}$ & $\begin{array}{l}\text { By request: z.r.yang@ex.ac.uk } \\
\text { By request: jebeliza@usp.br } \\
\text { http://wwwalt.med-rz.uniklinik- } \\
\text { saarland.de/med_fak/ } \\
\text { humangenetik/software/ } \\
\text { index.html }\end{array}$ \\
\hline PEPS $^{40}$ & Training set: 11 substrates & P4-P2' & No & Caspase-3 only & $\begin{array}{l}\text { By request: } \\
\text { thomas.reineckel@unikinik- } \\
\text { freidburg.de }\end{array}$ \\
\hline PoPS $^{38}$ & $\begin{array}{l}\text { Inherent specificity, plus } \\
\text { manual weighting }\end{array}$ & $\mathrm{P} 4-\mathrm{P} 1^{\prime}$ & $\begin{array}{l}\text { Predicts buried residues, } \\
\text { secondary structure, PEST } \\
\text { sequences }\end{array}$ & Caspases 1-9 & $\begin{array}{l}\text { http:// } \\
\text { pops.csse.monash.edu.au/ } \\
\text { index.html }\end{array}$ \\
\hline
\end{tabular}

${ }^{\mathrm{a}}$ Training sets use protein cleavage sites from the literature. Inherent specificity is from combinatorial scanning using synthetic peptides. ${ }^{\mathrm{b}} \mathrm{Pan}$-caspase signifies that individual caspase predictions are not supported.

substrates remain elusive, although they may explain the discrepancies between cleavage consensuses and those found in cell-based experiments. ${ }^{18-20}$ Interestingly, a number of caspase-3 substrates are processed at noncanonical sites. ${ }^{18,21,22}$ These findings imply that exosites in caspases recognize motifs in their substrates in addition to, and in some cases superseding, the specificity requirements derived from studies with short synthetic peptides.

Although exosite interactions of caspases and substrates remain elusive, members of the inhibitor of apoptosis protein (IAP) family clearly bind caspases outside the active site through association with the neo $\mathrm{N}$-terminus of the caspase generated by cleavage of the interchain linker. ${ }^{23-27}$ In contrast to the many structures solved at the atomic level with synthetic substrate analogs bound to caspases, the only structure of a natural substrate bound is that of the viral caspase inhibitor p35 to caspase-8, which is trapped as a covalent adduct. ${ }^{28}$ Interestingly, p35 contains a loop that interacts with a face of caspase- 8 distant from the active site, possibly revealing an exosite interaction. ${ }^{29}$

The role of cleavage site conformation. Few structures have been solved for caspase substrates, and those that have reveal that the site of cleavage is in a disordered mobile loop that is not visualized with any degree of certainty in the crystal structures or NMR models. Thus, Bid (a caspase-8 substrate $^{30,31}$ ), DFF45/inhibitor of caspase-activated DNAse (ICAD) (a caspase-3 substrate ${ }^{32,33}$ ) and procaspase-7 (a caspase-8 and 9 substrate ${ }^{34,35}$ ), all confirmed in vivo substrates for caspases, lack any detail of the requirements for cleavage. All cleavage sites map to highly flexible and exposed linkers that may be accessible to caspases without the need for exosite interactions.

Indeed, unstructured extended loops seem to be common features of natural protein substrate cleavage sites for proteases in general. ${ }^{36}$ Are these flexible extended loops functioning simply to present the cleavage site in the most accessible way, or do they have an additional unappreciated function? Although any type of induced folding of these mobile loops has yet to be shown for caspase-substrate interaction, the local structure of the cleavage site is likely a vital requirement for proteolysis. An example showing the importance of solvent-exposed, disordered loops that present nonoptimal cleavage sequences is the case of pro-IL-1b. Heat-denatured pro-IL-1b was cleaved by caspase-1 as efficiently as the folded precursor. ${ }^{37}$

Cleavage site predictions. Current efforts at predicting caspase cleavage sites in proteins are focused on prediction through searching for potential sites in translated genomic data using inherent substrate specificity models. ${ }^{38-40}$ Others have developed caspase prediction software and neural network analysis that uses examples of known cleavage sites (training sets) to infer preferred cleavage site amino acids, ${ }^{41,42}$ but here the major question must be the quality of the training set (Table 1). The uncontrolled variable is the quality of the data used to define the training set, which is often based simply on literature reports. The assumption that all literature reports give equivalent confidence is, of course, problematic. Biological events are not binary. One does not have binding or no binding, phosphorylation or no phosphorylation, proteolysis or no proteolysis in vivo. Biological events are analog, and what really counts are relative rates (or efficiencies) of interaction and the vast majority of literature reports have yet to come to grips with this. The drive to understand apoptosis and identify caspase substrates will test the efficiency and usefulness of these tools as their predictions are evaluated.

More direct methods for discovering natural caspase substrates include adding active recombinant caspases to proteins obtained by expression of mRNAs. ${ }^{43}$ This type of method, which was used to discover Bid (see below) as a caspase- 8 substrate, ${ }^{43}$ has the advantage that entire transcriptomes can be covered, but suffers from the problem that many proteins will lack post-translational modifications required for their proper folding. Other methods involve proteomics-based analysis of cell fractions cleaved by recombinant caspases, ${ }^{44}$ and perhaps most appropriately by chemical modification of proteins generated by bone fide apoptotic events to reveal endogenous sites. ${ }^{45}$ The latter method, based on identification of neo $\mathrm{N}$-termini which map to the C-terminal cleavage product of proteolysis, has the advantage of finding the products of proteolysis produced in vivo. Related methodologies are very promising for finding out 
what caspases (or other proteases for that matter) actually do cleave in vivo, rather than what they may cleave in vitro. Yet in the end, all of these methods require validation, so that one can distinguish an innocent bystander from an important culprit.

\section{Natural Protein Substrates}

It was suggested in the early days of caspase investigation that the sum total of the many proteolytic events that constitute cleavage of endogenous proteins by caspases defines the complex cellular morphology known as apoptosis. ${ }^{46,47}$ To test this prediction, we would need to separate the cleavage events that cause apoptotic function/morphology from those innocent bystander events that are inevitable given the complexity of animal proteomes. It is not so easy to do this in a scientifically rigorous manner. Owing to the continual proliferation in number of reported proteins that are cleaved by caspases, we do not list them in this review, but refer readers to a Web-based resource that continually updates them. This server is part of the bioinformatics and outreach component of the Center on Proteolytic Pathways, a National Institutes of Health Roadmap Initiative, and is located at http://cutdb.burn ham.org/. The resource covers many human proteases, and caspases and their substrates can be accessed by using the menus. In principle we could think of reported caspase substrates as fitting into five categories, somewhat related to the confidence in their assignment as naturally occurring events:

- Proteins demonstrated to be cleaved by recombinant caspases in a purified system (preferably including rate $k_{\text {cat }} / K_{\mathrm{m}}-$ calculations).

- Cleavage products identified in cells.

- Cleavage site mutants generated, showing abrogation of cleavage.

- Cleavage site mutants introduced into deficient cells.

- Cleavage site mutants introduced transgenically into whole animals.

Obviously one would want to go as far down this list as possible to confirm the existence and biological importance of a putative event, but this is challenging for many proteins. Indeed, only a handful of the hundreds of reported events have been confirmed to be of significance in vivo, and we highlight a few categories below. It is important to remember that, just as for any post-translational modification, caspase cleavage can have a gain-of-function or loss-of-function consequence for the protein. For loss-of-function events one would expect a substantial amount of the protein to be cleaved, but for a gain-of-function there need not, in principle, be more than a small portion of the total molecules cleaved to allow the product(s) to signal. Currently, there is virtually no knowledge of how much cleavage of any caspase substrate there should be for a biologic or pathologic outcome. Ultimately, in the transgenic models where a caspase cleavage site has been replaced by a noncleavable one, usually an Asp-Ala mutation, there is the formal, albeit lowlevel, likelihood that a function of the protein other than proteolysis has been modified by the mutation.

\section{Gain-of-function}

Executioner procaspase zymogens. Given that apoptosis is, minimally, a two-step pathway in which apical (initiator) caspases such as caspase-8, -9 and -10 activate the zymogens of executioner caspases-3 and -7, the best known caspase substrates are themselves caspases reviewed by Fuentes-Prior and Salvesen. ${ }^{48}$ In fact, the zymogens of caspase- 3 and -7 are great substrates of the initiator caspases, with $k_{\mathrm{cat}} / K_{\mathrm{m}}$ values in the range of $10^{5} \mathrm{M}^{-1} \mathrm{~s}^{-1} .49$ Their cleavage sites fit the caspase- 8 and -9 consensus, ${ }^{8}$ and cleavage has a very clear gain-of-function role, namely to activate the proteases for proteolysis of downstream substrates. The caspase- 8 and -9 cleavage site within procaspase-7, IQAD $198 \downarrow S$ is contained in an extended loop, known as the interchain connector, that is not resolved in the crystal structures of the zymogen. ${ }^{34,35}$ Cleavage in this unstructured loop generates small conformational changes in the protein that greatly enhance stability of the active site of caspase-7, thereby propagating the apoptotic signal.

Bid. Caspase- 8 cleaves Bid about as efficiently as it cleaves procaspase- 3 in vitro. ${ }^{50}$ The solution structures of Bid reveals that the caspase-8 cleavage site, LQTD $60 \downarrow$, maps to an unusually long, highly flexible loop. ${ }^{30,31}$ Therefore, the Asp $_{60} \downarrow$ Gly scissile bond is readily targeted by caspase-8 with the $\mathrm{P}_{4}-\mathrm{P}_{1}{ }^{\prime}$ residues conforming to the inherent substrate specificity (see above). An argument for an exosite interaction comes from the observation that caspase-8 does not cleave a second site found in the same loop $I Q A D_{75} \downarrow S$. This site appears accessible, as it is cleaved by granzyme $\mathrm{B}$, with essentially identical gain-of-function properties with respect to promoting apoptosis. ${ }^{51}$ Possible exosite-mediated interactions preferentially guide the Asp $_{60} \downarrow$ Gly site of Bid into the active site cleft of caspase-8, or conversely steer the caspase-away from the $A s p_{75} \downarrow$ Ser site. Recombinant variants of Bid with mutated or swapped cleavage sites might help clarify this point. It is strangely still unclear why cleavage of Bid activates it for participation in the Bax/Bak-mediated mitochondrial pathway. The cleaved loop lies between helices 2 and 3 and the possibility that cleavage releases helix 2 to expose helix 3 (the $\mathrm{BH} 3$ domain) seems improbable given that no significant differences in conformation, judged by NMR or gel filtration, occur following caspase-8 cleavage of Bid. ${ }^{30}$ Perplexingly, despite the dozens of publications implicating Bid as an important sensor of extrinsic pathway death stimuli in vitro, replacement of $\mathrm{Bid}-/-$ mice with a transgene containing the cleavage site mutant LQTA $60 \downarrow G$ had little, if any, negative impact on apoptotic induction by a number of stimuli, ${ }^{52}$ raising some doubts about the importance of the cleavage for apoptosis in vivo.

$R B$. Retinoblastoma-associated protein $(R B)$ is a tumor suppressor that inhibits cell cycle progression into S-phase by inhibiting transcription from E2F promoters. Caspases cleave in the C-terminal region of RB at $D E A D_{886} \downarrow G$ as a consequence of apoptosis through the extrinsic pathway, but not intrinsic pathway, resulting in its destabilization and degradation. ${ }^{53}$ In support of the hypothesis that RB must be degraded for tumor necrosis factor receptor (TNFR)-Imediated apoptosis, germline replacement of the caspase cleavage site with the uncleavable sequence $D_{E A A_{886} \downarrow G}$ 
protects fibroblasts against TNFR-I-induced apoptotic signaling in vitro, and intestinal cells from endotoxic shock in vivo. This deficiency can be overcome through ligation and signaling through both TNFR-I and II, suggesting an alternative mechanism for RB degradation. ${ }^{53}$ So it seems that $R B$ is a sentinel of survival that must be inactivated or removed for progression of apoptosis through the extrinsic pathway in mouse intestine. It remains to be determined which RB function is preventing apoptosis, and how caspase cleavage of $\mathrm{RB}$ precipitates its degradation.

$I A P$ binding motifs. Most of the gain- or loss-of-function events caused by caspase cleavage are related to changes in the intrinsic properties of the protein being cleaved. For example, one may have removal of a regulatory domain, or zymogen activation, or dissociation of active domains. However, there is a function for caspase cleavage where the $\mathrm{N}$-terminal neoepitope generated by proteolysis may itself have a purpose. The best examples are the epitopes generated in caspases- 7 and -9 that allow recruitment of IAPs (see above), and the motifs are therefore known as IAP binding motifs (IBMs). Therefore, it is interesting to ponder whether IBMs are generated as a consequence of the cleavage of other proteins. Indeed, this possibility has been investigated, ${ }^{54}$ but the resulting proteins have rather weak $K_{\mathrm{d}} \mathrm{s}$, much too weak to be physiologically significant. However, if the target proteins are oligomeric like the caspases (and second mitochondrial activator of caspases/ direct IAP binding protein with low pl (Smac/DIABLO), and HtrA2/Omi - two other IBM proteins with IAP-antagonist function) then avidity is enhanced and $K_{d} s$ reach the physiologic range because of the mode of interaction with IAPs. ${ }^{55}$ With this stipulation the products of caspase cleavage, providing the neoepitopes generated have an IBM sequence, can in theory at least become IAP antagonists and promote apoptosis in a feed-forward manner by competing with caspases- 7 and -9 themselves for binding to IAPs, thereby removing the IAP block to caspase activity.

\section{Loss-of-function}

DFF45/ICAD. We classify this as a loss-of-function event because, even though the outcome is activation of a nuclease, the reason is loss of its chaperone/inhibitor. The nuclease responsible for double-strand cleavages in apoptosis, DFF40/CAD, exists in a tight noncovalent complex with its endogenous chaperone/inhibitor, DFF45/ ICAD. This complex is disrupted when caspase-3 cleaves ICAD at positions $D T_{117} \downarrow S$ and $D E V D_{224} \downarrow T$, located between domains D1 and D2, and D2 and D3, respectively. These events allow the free nuclease to dimerize into the catalytically competent form. ${ }^{32,33,56,57}$ As with Bid, both cleavage sites map to presumably highly flexible and exposed linkers. The crystal structure of activated CAD features a $\mathrm{Zn}^{2+}$-mediated dimer. ${ }^{33}$ The central D2 domain of ICAD is critical for the inhibitory activity, and it is thought that inhibition is relieved after caspase-cleavage due to the low affinity of the isolated domain for the enzyme. Thus, the relief from ICAD inhibition basically relies not on destruction of the inhibitor in the manner that the proteasome destroys the NK$\kappa \mathrm{B}$ inhibitor $\mathrm{I}_{\kappa} \mathrm{B}$, but rather on limited cleavages that loosen up the structure of ICAD just enough to allow displacement by another unit of CAD to allow dimerization of the catalytic nuclease.

Poly(ADP-ribose) polymerase. Poly(ADP-ribose) polymerase (PARP) is an abundant nuclear protein that catalyses poly(ADP-ribose) ligation to acceptor proteins, including itself, in response to DNA strand breaks. PARP cleavage by caspases-3 and -7 at the DEVD $213 \downarrow$ G site bisects a bipartite nuclear localization signal, generating a form that cannot synthesize ADP-ribose polymers in response to damaged DNA. ${ }^{58}$ PARP was one of the first identified examples of a substrate processed by an executioner caspase much more efficiently than the inflammatory caspase-1. ${ }^{59,60}$ The PARP cleavage site perfectly matches the substrate specificity of executioner caspases-3 and -7. Interestingly, secondary interactions seem to be at work because caspase-7, which is less catalytically active than caspase-3 on short synthetic substrates, processes PARP molecules modified with long, branched poly(ADP-ribose) chains more efficiently than its close homolog caspase-3. The enhanced activity results from interactions between the large subunit of caspase-7 with poly(ADP-ribose) that are not present with caspase-3, ${ }^{61}$ hinting at an exosite interaction. Mice expressing a transgenic PARP caspase cleavage site mutant $\left(D V_{213} \downarrow G\right)$ develop normally, but resist endotoxic shock and intestinal and renal ischemia-reperfusion in vivo, ${ }^{62}$ indicating that failure to cleave the protein has similar tissue-restricted effects to mice defective in caspasemediated RB cleavage (see above).

\section{Feedback regulation}

Caspase-9. The initiator caspase of the postmitochondrial intrinsic pathway, caspase- 9 , is cleaved at two sites, $P P_{315} \downarrow A$ and $D Q L D_{330} \downarrow A$, in the interchain linker region. Although these cleavages are dispensable for activation, ${ }^{63}$ they have an important role in regulating apoptosis. Cleavage at $\mathrm{PEPD}_{315} \downarrow \mathrm{A}$ produces a neo- $\mathrm{N}$ terminal epitope, the sequence ${ }_{316} \mathrm{ATPF}$, which is essential for inhibition of caspase- 9 by its endogenous regulator $X$ linked IAP (XIAP). ${ }^{23}$ This cleavage is produced by caspase- 9 itself, and seems to dampen apoptotic responses through interruption of the pathway at this crucial stage. Cleavage at $D L D_{330} \downarrow A$, mediated by executioner caspases, removes the inhibitory interaction and renders caspase- 9 refractory to inhibition by XIAP. This event therefore promotes apoptosis, and can be thought of as a feedback where caspase-3 activates the proteolytic potential of caspase-9. Importantly, caspase- 3 does this not by activating caspase- 9 directly, but by removing the neoepitope exosite required for caspase- 9 inhibition, and therefore this event can be thought of as 'derepression' rather than 'activation'.

\section{Neuropathogenic substrates}

Huntingtin. Huntington's disease is a neurodegenerative disorder with characteristic accumulation of $\mathrm{N}$-terminal Huntingtin (HTT) fragments in neuronal nuclei. Several caspase cleavage sites are located downstream of the characteristic poly-glutamine sequence whose expansion results in disease pathology. Transgenic mice expressing HTT with mutated potential caspase cleavage sites indicate 
one of these, the so-called caspase- 6 cleavage site located at ILVD $586 \downarrow$, is critical for generating a toxic HTT fragment. Transgenic mice expressing a ILVA I $_{56} \downarrow G$ cleavage site mutant are resistant to excitotoxic neuronal death. ${ }^{64}$ Although the function of HTT is unknown, overexpression of wild-type HTT has shown a protective phenotype under models of neuronal stress. ${ }^{65}$ Significantly, caspase cleavage of HTT is reported to not only remove HTT's protective function, but also generates a toxic byproduct that sensitizes neurons to further stressors such as excitotoxic stimulation. The mechanism of neurotoxicity in unknown, as is the nature and origin of the caspase activity, which of course must be generated somehow to produce the toxic fragment.

$A P P$. The action of the proteolytic enzymes beta and gamma secretases excises a partially membrane spanning fragment, $\beta$-amyloid $(\mathrm{A} \beta)$, from the amyloid precursor protein (APP). APP is additionally cleaved in its cytosolic tail at VEVD ${ }_{664} \downarrow A$ by caspases, and this fragment (C31) is associated with cell toxicity. ${ }^{66}$ Investigation of a transgenic mouse model for Alzheimer disease expressing human APP with the caspase cleavage site mutated to VEVA $\mathrm{V}_{664} \downarrow A$ shows protection from neuronal death and astrogliosis compared to the unmutated disease-causing variant of APP, while maintaining comparable levels of soluble and deposited $A \beta \quad 1-40$ and $1-42 .^{67}$ This evidence points to $\mathrm{C} 31$ as a neurotoxic insult in Alzheimer disease and not $\mathrm{A} \beta$, however, this does not negate the amyloid hypothesis as $\mathrm{A} \beta$ may induce cytoplasmic cleavage of APP by caspases to form the effector C31. So far, as for HTT (see above) it is unclear what caspase is cleaving APP to form C31, or exactly what the context is for caspase activation. It is also unknown how C31 accumulates in the nucleus and how it causes neuronal death.

\section{Strengthening Substrate Foundations}

Cleavage of caspase substrates is central to the process of apoptosis. Therefore, significant effort has gone into understanding how caspases recognize their substrates, what the critical substrates are, and how substrate cleavage leads to the apoptotic phenotype. The role of primary structure in caspase substrate cleavage is well understood from studies using positional scanning peptide libraries as well as accumulating empirical evidence from annotated substrate cleavage sites. Accordingly, several efforts have been made to apply this knowledge for caspase substrate prediction, however, the strength of the predictions are largely unvalidated due to a scarcity of experimentation. Caspase substrate prediction may be improved through insights into the role of secondary structure in substrate cleavage, but only a small number of cleavage site structures are currently available. Nevertheless, these examples suggest that unstructured extended loops are preferred conformations for caspase driven proteolysis. Although the list of annotated caspase substrates continues to increase, the majority of candidates lack functional evidence linking cleavage to a role in apoptosis. Rigorous investigation of cleavage site mutants in cells and animals will purge 'bystander' substrates from the list of relevant caspase substrates. By cleaning out the bystanders, we will gain a more realistic understanding of how caspases effect apoptotic cell death.
1. Alnemri ES, Livingston DJ, Nicholson DW, Salvesen G, Thornberry NA, Wong WW et al. Human ICE/CED-3 protease nomenclature. Cell 1996; 87: 171.

2. Odake S, Kam CM, Narasimhan L, Poe M, Blake JT, Krahenbuhl O et al. Human and murine cytotoxic $T$ lymphocyte serine proteases: subsite mapping with peptide thioester substrates and inhibition of enzyme activity and cytolysis by isocoumarins. Biochem USA 1991; 30: 2217-2227.

3. Harris JL, Backes BJ, Leonetti F, Mahrus S, Ellman JA, Craik CS. Rapid and general profiling of protease specificity by using combinatorial fluorogenic substrate libraries. Proc Natl Acad Sci USA 2000; 97: 7754-7759.

4. Stennicke HR, Renatus M, Meldal M, Salvesen GS. Internally quenched fluorescent peptide substrates disclose the subsite preferences of human caspases 1, 3, 6, 7 and 8 . Biochem J 2000; 350: 563-568.

5. Hawkins CJ, Yoo SJ, Peterson EP, Wang SL, Vernooy SY, Hay BA. The Drosophila caspase DRONC cleaves following glutamate or aspartate and is regulated by DIAP1, HID, and GRIM. J Biol Chem 2000; 275: 27084-27093.

6. Vercammen D, van de Cotte B, De Jaeger G, Eeckhout D, Peter Casteels P, Vandepoele K et al. Type-Il metacaspases Atmc4 and Atmc9 of Arabidopsis thaliana cleave substrates after arginine and lysine. J Biol Chem 2004; 279: 45329-45336.

7. Chen JM, Rawlings ND, Stevens RA, Barrett AJ. Identification of the active site of legumain links it to caspases, clostripain and gingipains in a new clan of cysteine endopeptidases. FEBS Lett 1998; 441: 361-365.

8. Thornberry NA, Rano TA, Peterson EP, Rasper DM, Timkey T, Garcia-Calvo M et al. A combinatorial approach defines specificities of members of the caspase family and granzyme B. Functional relationships established for key mediators of apoptosis. J Biol Chem 1997; 272: 17907-17911.

9. Talanian RV, Quinlan C, Trautz S, Hackett MC, Mankovich JA, Banach D et al. Substrate specificities of caspase family proteases. J Biol Chem 1997; 272: 9677-9682.

10. Kato D, Boatright KM, Berger $A B$, Nazif T, Blum G, Ryan $C$ et al. Activity-based probes that target diverse cysteine protease families. Nature Chem Biol 2005; 1: 33-38.

11. Faleiro L, Kobayashi R, Fearnhead H, Lazebnik Y. Multiple species of CPP32 and Mch2 are the major active caspases present in apoptotic cells. EMBO J 1997; 16: 2271-2281.

12. Denault JB, Salvesen GS. Human caspase-7 activity and regulation by Its $\mathrm{N}$-terminal peptide. J Biol Chem 2003; 278: 34042-34050.

13. Read SH, Baliga BC, Ekert PG, Vaux DL, Kumar S. A novel Apaf-1-independent putative caspase-2 activation complex. J Cell Biol 2002; 159: 739-745.

14. Tu S, McStay GP, Boucher LM, Mak T, Beere HM, Green DR. In situ trapping of activated initiator caspases reveals a role for caspase-2 in heat shock-induced apoptosis. Nat Cell Biol 2006; 8: 72-77.

15. Bode W, Brandstetter $\mathrm{H}$, Mather T, Stubbs MT. Comparative analysis of haemostatic proteinases: structural aspects of thrombin, factor $\mathrm{Xa}$, factor IXa and protein $\mathrm{C}$. Thromb Haemost 1997; 78: 501-511.

16. Lopez-Otin C, Overall CM. Protease degradomics: a new challenge for proteomics. Nat Rev Mol Cell Biol 2002; 3: 509-519.

17. Germain D, Dumas F, Vernet T, Bourbonnais Y, Thomas DY, Boileau G. The pro-region of the Kex2 endoprotease of Saccharomyces cerevisiae is removed by self-processing. FEBS Lett 1992; 299: 283-286.

18. Nicholson DW. Caspase structure, proteolytic substrates, and function during apoptotic cell death. Cell Death Differ 1999; 6: 1028-1042.

19. Thornberry NA, Lazebnik Y. Caspases: enemies within. Science 1998; 281: 1312-1316.

20. Stennicke HR, Salvesen GS. Caspases - controlling intracellular signals by protease zymogen activation. Biochim Biophys Acta 2000; 1477: 299-306.

21. Earnshaw WC, Martins LM, Kaufmann SH. Mammalian caspases: structure, activation, substrates, and functions during apoptosis. Annu Rev Biochem 1999; 68: 383-424.

22. Fischer U, Janicke RU, Schulze-Osthoff K. Many cuts to ruin: a comprehensive update of caspase substrates. Cell Death Differ 2003; 10: 76-100.

23. Srinivasula SM, Hegde R, Saleh A, Datta P, Shiozaki E, Chai J et al. A conserved XIAPinteraction motif in caspase- 9 and Smac/DIABLO regulates caspase activity and apoptosis. Nature 2001; 410: 112-116.

24. Shiozaki EN, Chai J, Rigotti DJ, Riedl SJ, Li P, Srinivasula SM et al. Mechanism of XIAPmediated inhibition of caspase-9. Mol Cell 2003; 11: 519-527.

25. Scott FL, Denault JB, Riedl SJ, Shin H, Renatus M, Salvesen GS. XIAP inhibits caspase-3 and -7 using two binding sites: evolutionarily conserved mechanism of IAPs. EMBO J2005; 24: 645-655.

26. Tenev $\mathrm{T}$, Zachariou A, Wilson R, Ditzel M, Meier P. IAPs are functionally non-equivalent and regulate effector caspases through distinct mechanisms. Nat Cell Biol 2005; 7: 70-77.

27. Eckelman BP, Salvesen GS. The human anti-apoptotic proteins CIAP1 and cIAP2 bind but do not inhibit caspases. J Biol Chem 2006; 281: 3254-3260.

28. Xu G, Cirilli M, Huang Y, Rich RL, Myszka DG, Wu H. Covalent inhibition revealed by the crystal structure of the caspase-8/p35 complex. Nature 2001; 410: 494-497.

29. Fisher AJ, Cruz W, Zoog SJ, Schneider CL, Friesen PD. Crystal structure of baculovirus P35: role of a novel reactive site loop in apoptotic caspase inhibition. EMBO J 1999; 18: 2031-2039.

30. Chou JJ, Li H, Salvesen GS, Yuan J, Wagner G. Solution structure of BID, an intracellular amplifier of apoptotic signaling. Cell 1999; 96: 615-624.

31. McDonnell JM, Fushman D, Milliman CL, Korsmeyer SJ, Cowburn D. Solution structure of the proapoptotic molecule BID: a structural basis for apoptotic agonists and antagonists. Cell 1999; 96: 625-634. 
32. Otomo T, Sakahira H, Uegaki K, Nagata S, Yamazaki T. Structure of the heterodimeric complex between CAD domains of CAD and ICAD. Nat Struct Biol 2000; 7: 658-662.

33. Woo EJ, Kim YG, Kim MS, Han WD, Shin S, Robinson H et al. Structural mechanism for inactivation and activation of CAD/DFF40 in the apoptotic pathway. Mol Cell 2004; 14: 531-539.

34. Chai J, Wu Q, Shiozaki E, Srinivasula SM, Alnemri ES, Shi Y. Crystal structure of a procaspase-7 zymogen. Mechanisms of activation and substrate binding. Cell 2001; 107: 399-407.

35. Riedl SJ, Fuentes-Prior P, Renatus M, Kairies N, Krapp R, Huber R et al. Structural basis for the activation of human procaspase-7. Proc Natl Acad Sci USA 2001; 98 14790-14795.

36. Hubbard SJ, Campbell SF, Thornton JM. Molecular recognition. Conformational analysis of limited proteolytic sites and serine proteinase protein inhibitors. J Mol Biol 1991; 220: 507-530.

37. Sleath PR, Hendrickson RC, Kronheim SR, March CJ, Black RA. Substrate specificity of the protease that processes human interleukin-1 $\beta$. J Biol Chem 1990; 265: 14526-14528.

38. Boyd SE, Pike RN, Rudy GB, Whisstock JC, Garcia de la Banda M. PoPS: a computational tool for modeling and predicting protease specificity. J Bioinform Comput Biol 2005; 3 551-585.

39. Backes C, Kuentzer J, Lenhof HP, Comtesse N, Meese E. GraBCas: a bioinformatics too for score-based prediction of Caspase- and granzyme B-cleavage sites in protein sequences. Nucleic Acids Res 2005; 33: W208-W213.

40. Lohmuller T, Wenzler D, Hagemann S, Kiess W, Peters C, Dandekar T et al. Toward computer-based cleavage site prediction of cysteine endopeptidases. Biol Chem 2003; 384: 899-909.

41. Garay-Malpartida HM, Occhiucci JM, Alves J, Belizario JE. CaSPredictor: a new computerbased tool for caspase substrate prediction. Bioinformatics 2005; 21 (Suppl 1): i169-i176.

42. Yang ZR. Prediction of caspase cleavage sites using Bayesian bio-basis function neural networks. Bioinformatics 2005; 21: 1831-1837.

43. Li H, Zhu H, Xu CJ, Yuan J. Cleavage of BID by caspase 8 mediates the mitochondrial damage in the Fas pathway of apoptosis. Cell 1998; 94: 491-501.

44. Ricci JE, Munoz-Pinedo C, Fitzgerald P, Bailly-Maitre B, Perkins GA, Yadava N et al. Disruption of mitochondrial function during apoptosis is mediated by caspase cleavage of the p75 subunit of complex I of the electron transport chain. Cell 2004; 117: 773-786.

45. Van Damme P, Martens L, Van Damme J, Hugelier K, Staes A, Vandekerckhove J et al. Caspase-specific and nonspecific in vivo protein processing during Fas-induced apoptosis. Nat Methods 2005; 2: 771-777.

46. Martin SJ, Green DR. Protease activation during apoptosis: death by a thousand cuts? Cell 1995; 82: 349-352.

47. Salvesen GS, Dixit VM. Caspases: intracellular signaling by proteolysis. Cell 1997; 91 443-446.

48. Fuentes-Prior P, Salvesen GS. The protein structures that shape caspase activity, specificity, activation and inhibition. Biochem J 2004; 384: 201-232.

49. Stennicke HR, Jurgensmeier JM, Shin H, Deveraux Q, Wolf BB, Yang X et al. Pro-caspase3 is a major physiologic target of caspase-8. J Biol Chem 1998; 273: 27084-27090.

50. Bossy-Wetzel E, Green DR. Caspases induce cytochrome $c$ release from mitochondria by activating cytosolic factors. J Biol Chem 1999; 274: 17484-17490.

51. Barry M, Heibein JA, Pinkoski MJ, Lee SF, Moyer RW, Green DR et al. Granzyme B shortcircuits the need for caspase 8 activity during granule-mediated cytotoxic T-lymphocyte killing by directly cleaving Bid. Mol Cell Biol 2000; 20: 3781-3794.
52. Sarig R, Zaltsman $Y$, Marcellus RC, Flavell R, Mak TW, Gross A. BID-D59A is a potent inducer of apoptosis in primary embryonic fibroblasts. J Biol Chem 2003; 278 10707-10715.

53. Chau BN, Borges HL, Chen TT, Masselli A, Hunton IC, Wang JY. Signal-dependent protection from apoptosis in mice expressing caspase-resistant Rb. Nat Cell Biol 2002; 4 757-765.

54. Hell K, Saleh M, Crescenzo GD, O'Connor-McCourt MD, Nicholson DW. Substrate cleavage by caspases generates protein fragments with $\mathrm{Smac} /$ Diablo-like activities. Cell Death Differ 2003; 10: 1234-1239.

55. Huang Y, Rich RL, Myszka DG, Wu H. Requirement of both the BIR2 and BIR3 domains for the relief of XIAP-mediated caspase inhibition by Smac. J Biol Chem 2003.

56. Sakahira $H$, Enari $M$, Nagata $S$. Cleavage of CAD inhibitor in CAD activation and DNA degradation during apoptosis. Nature 1998; 391: 96-99.

57. Liu X, Zou H, Widlak P, Garrard W, Wang X. Activation of the apoptotic endonuclease DFF40 (caspase-activated DNase or nuclease). Oligomerization and direct interaction with histone H1. J Biol Chem 1999; 274: 13836-13840.

58. Lazebnik YA, Kaufmann SH, Desnoyers S, Poirier GG, Earnshaw WC. Cleavage of poly(ADP-ribose) polymerase by a proteinase with properties like ICE. Nature 1994; 371 346-347.

59. Gu Y, Sarnecki C, Aldape RA, Livingston DJ, Su MSS. Cleavage of poly(ADP-ribose) polymerase by interleukin-1-beta converting enzyme and its homologs TX and Nedd-2. J Bio Chem 1995; 270: 18715-18718.

60. Tewari M, Quan LT, O'Rourke K, Desnoyers S, Zeng Z, Beidler DR et al. Yama/CPP32beta, a mammalian homolog of CED-3, is a CrmA-inhibitable protease that cleaves the death substrate poly(ADP-ribose) polymerase. Cell 1995; 81: 801-809.

61. Germain M, Affar EB, D'Amours D, Dixit VM, Salvesen GS, Poirier GG. Cleavage of automodified Poly(ADP-ribose) polymerase during apoptosis. Evidence for involvement of caspase-7. J Biol Chem 1999; 274: 28379-28384.

62. Petrilli V, Herceg Z, Hassa PO, Patel NS, Di Paola R, Cortes U et al. Noncleavable poly(ADP-ribose polymerase-1 regulates the inflammation response in mice. J Clin Invest 2004; 114: 1072-1081.

63. Stennicke HR, Deveraux QL, Humke EW, Reed JC, Dixit VM, Salvesen GS. Caspase- 9 can be activated without proteolytic processing. J Biol Chem 1999; 274: 8359-8362.

64. Graham RK, Deng Y, Slow EJ, Haigh B, Bissada N, Lu G et al. Cleavage at the caspase-6 site is required for neuronal dysfunction and degeneration due to mutant Huntingtin. Cell 2006; 125: 1179-1191.

65. Zhang Y, Li M, Drozda M, Chen M, Ren S, Mejia Sanchez RO et al. Depletion of wild-type Huntingtin in mouse models of neurologic diseases. J Neurochem 2003; 87 101-106.

66. Lu C, Fu W, Salvesen GS, Mattson MP. Direct cleavage of AMPA receptor subunit GluR and suppression of AMPA currents by caspase-3: implications for synaptic plasticity and excitotoxic neuronal death. Neuromol Med 2002; 1: 69-79.

67. Galvan V, Gorostiza OF, Banwait S, Ataie M, Logvinova AV, Sitaraman S et al. Reversal of Alzheimer's-like pathology and behavior in human APP transgenic mice by mutation of Asp664. Proc Natl Acad Sci USA 2006; 103: 7130-7135.

68. Schweizer A, Briand C, Grutter MG. Crystal structure of caspase-2, apical initiator of the intrinsic apoptotic pathway. J Biol Chem 2003; 278: 42441-42447.

69. Schecter I, Berger M. On the size of the active site in proteases. Biochem Biophys Res Commun 1967; 27: 157-162. 